PATRYCJA CHRUŚCIEL

ORCID: 0000-0003-0345-1280

Université de Wrocław

patrycja.chrusciel@uwr.edu.pl

\title{
PAYS, BASTION ET FORTERESSE CATHOLIQUE? À PROPOS DE LA CONSTRUCTION DU SENS SOCIAL DU NOM PROPRE POLOGNE DANS LE DISCOURS DE LA PRESSE ÉCRITE
}

\section{INTRODUCTION}

Cette étude a pour objet le sens social du nom propre Pologne étudié dans le contexte de ses emplois avec l'adjectif catholique. Nos analyses font partie d'un projet plus large — notre thèse de doctorat — visant à décrire, selon la méthodologie proposée par l'analyse linguistique du discours (désormais ALD) ${ }^{1}$, le sens social du nom propre Pologne construit dans le discours ${ }^{2}$ de la presse quotidienne, pendant une période de quinze ans comprise entre l'adhésion de la Pologne à l'Union Européenne en 2004 et l'époque actuelle. Selon les principes de l'ALD $^{3}$ que nous adoptons dans notre étude, le sens social est déterminé par

${ }^{1}$ Les références aux études propres à ce courant sont données au fil de cette étude.

${ }^{2}$ En prenant pour objet de notre étude le sens du toponyme Pologne, nous inscrivons notre projet dans le cadre des travaux consacrés à la sémantique discursive des noms propres $(c f$. G. Cislaru, Étude sémantique et discursive du nom de pays dans la presse française avec la référence à l'anglais, au roumain et au russe, thèse de doctorat en science du langage, sous la direction de B. Bosredon et S. Moirand, Université Paris 3 - Sorbonne Nouvelle, 2005 ; " Le nom propre en discours ». Les Carnets du Cediscor 11, M. Lecolle, M.-A. Paveau, S. Reboul-Touré (dir.), 2009).

${ }^{3}$ Par l'analyse linguistique du discours, on entend l'ensemble des recherches proposées dans ce domaine à partir des années 1980-1990 (H. Grzmil-Tylutki, Francuska lingwistyczna teoria dyskursu, Universitas, Kraków 2010, p. 127). Cette approche s'inscrit dans la continuité des études 
un ensemble de traits lexico-discursifs (formant un profil lexico-discursif) propre à une unité linguistique. Il s'actualise dans un contexte socio-historique donné, au sein d'une communauté linguistique et pour les besoins de cette communauté. Il s'agit donc d'un sens intentionnel qui partage inévitablement le point de vue de la société dans laquelle il a été établi, d'un sens qui est une sorte d'interprétation du réel dans une perspective intersubjective valide ${ }^{4}$. Il est l'effet de l'activité de nomination effectuée par les médias, qui font un travail sémantique d'interprétation en nommant des événements, des phénomènes et des objets du monde réel. La nomination, livrant un point de vue anthropologique sur l'objet nommé ${ }^{5}$, crée une relation pratique entre le nom et celui-ci. Autrement dit, la nomination ne nomme pas l'objet « en soi » (comme le fait la dénomination), mais « pour nous $»^{6}$.

Dans notre étude, nous adhérons ainsi aux positions de recherches de la sémantique discursive conduites par Georgeta Cislaru ${ }^{7}$, qui montre dans son travail que le nom de pays est porteur de sens et que le lieu de sa construction est le discours de presse. Le nom propre, comme tout nom d'ailleurs, ne réfère qu'en discours : son actualisation en contexte sélectionne le référent déterminé par son potentiel sémantico-référentiel.

Le but de notre travail est de décrire l'apport de l'adjectif catholique dans le sens social du nom propre Pologne. Selon les dictionnaires consultés ${ }^{8}$, catho-

de l'École française d'analyse du discours (par ex. : D. Maldidier, « Le discours politique de la guerre d'Algérie : approche synchronique et diachronique », Langages 23, 1971, pp. 57-86 ; L. Guespin, « Problématique des travaux sur le discours politique », Langages 23, 1971, pp. 3-24 ; P. Sériot, Analyse du discours politique soviétique, Institut d'études slaves, Paris 1985, qui, dans les années 1960 et 1970, se concentraient sur le discours politique. La différence entre ces deux courants se trouve dans leur objet d'étude : à partir des années 1980-1990, les analystes du discours se sont orientés plutôt vers le discours médiatique.

L'analyse linguistique du discours confère au discours médiatique un rôle essentiel dans la construction des sens. Il s'agit d'un travail sémantique consistant en la nomination des événements réels et en la diffusion de ces noms qui, en circulant dans une société donnée, s'ancrent plus ou moins durablement dans la mémoire collective des lecteurs ( $c f$. S. Moirand, Les discours de la presse quotidienne : observer, analyser, comprendre, PUF, Paris 2007 ; M. Veniard, La nomination des événements dans la presse : essai de sémantique discursive, Presses Universitaires de FrancheComté, Besançon 2013). Les sens ainsi construits sont conçus comme des faits dynamiques relevant de déterminations historiques, culturelles, sociales et politiques ( $c f$. M. Lecolle, M. Veniard, O. Guérin, « Pour une sémantique discursive : propositions et illustrations », Langages 210, 2018, pp. 35-54).

${ }^{4}$ M. Veniard, op. cit., p. 24.

${ }^{5}$ Ibidem, p. 17.

${ }^{6}$ P. Siblot, « Nomination et production de sens : le praxème », Langages 127, 1997, pp. 38-55.

${ }^{7}$ G. Cislaru, op. cit.

${ }^{8}$ Catholique, [article dans :] Trésor de la Langue Française informatisé (désormais TLFi), $<$ http://atilf.atilf.fr/> [consulté le 28.08.2018] ; catholique, [article dans :] Le Petit Robert. Dictionnaire alphabétique et analogique de la Langue Française, J. Rey-Debove, A. Rey (dir.), Le Robert, Paris 2012, p. 370 ; catholique, [article dans :] Dictionnaire de français Larousse, $<$ http://larousse.fr/dictionnaires/francais/catholique/13810> [consulté le 10.09.2018]. 
lique est un adjectif relationnel qui se rapporte au substantif catholicisme. Il désigne quelqu'un ou quelque chose « qui se rapporte à l'Église romaine, qui lui est propre (par ex. doctrine, foi, religion catholique) » ou « qui professe la doctrine, la religion de l'Église catholique romaine, qui est placé sous son autorité (par ex. faculté, institut, université catholique) $»^{9}$.

Toujours selon les principes de $1^{\prime} A L D^{10}$, nous admettons que l'apport de cet adjectif au sens social du nom propre Pologne est déterminé par ses cooccurrences avec ce nom propre (axe syntagmatique) ainsi que par les reformulations, dans le réseau diaphorique, du SN ayant Pologne comme noyau et contenant l'adjectif catholique (axe textuel ou paradigmatique). L'étude du contexte syntagmatique et du contexte paradigmatique nous a conduits à établir une liste de reformulants formant un paradigme désignationnel ${ }^{11}$ dont les éléments ont la propriété de se rapporter au même référent.

Le corpus de textes de nos analyses est tiré de trois grands quotidiens nationaux : Le Monde (désormais LM), Le Figaro (LF) et Libération (L). Ce choix s'explique par le tirage des journaux en question ${ }^{12}$, par l'hétérogénéité de leur audience ${ }^{13}$, ainsi que par leur statut dit $«$ de référence ${ }^{14}$. Cependant, pour illustrer les tendances quantitatives des emplois étudiés, nous ne nous limiterons pas à ces seuls quotidiens. Nos analyses seront accompagnées de remarques sur les tendances quantitatives enregistrées dans toute la presse française ${ }^{15}$.

Pour recueillir le corpus d'exemples de notre travail, nous avons consulté la base de données Europresse dans laquelle nous avons lancé une recherche des occurrences contenant le nom propre Pologne et l'adjectif catholique(s) (dans un ordre indifférent) avec un maximum de cinq mots d'écart, dans des articles de presse parus entre le $1^{\mathrm{er}}$ mai 2004 (l'adhésion de la Pologne à l'Union européenne) et l'année $2018^{16}$. Nous avons extrait ensuite les occurrences dans lesquelles l'adjectif se rattache directement à ce nom propre ou, sur un mode qu'on peut dire indirect, entre

9 TLFi, définition du mot catholique.

10 M.-F. Mortureux, « Paradigmes désignationnels », Semen 8, 1993, <https://journals.openedition.org/semen/4132> [consulté le 11.09.2018].

11 Ibidem.

12 Le Figaro, Le Monde et Libération sont considérés comme des journaux de grande diffusion. En 2017, ils ont été diffusés respectivement à 308 000, 285000 et 75000 exemplaires par jour (source : Alliance pour les Chiffres de la Presse et des Médias, <http://www.acpm.fr> [consulté le 28.08.2018]).

${ }^{13}$ Cf. P. Eveno, La presse, Presses Universitaires de France, Paris 2016, pp. $46-47$.

${ }^{14}$ Cf. J.-M. Charon, La presse quotidienne, La Découverte, Paris 2013, pp. 36-37.

15 Par toute la presse française, nous entendons les titres disponibles dans la base de données Europresse. Le répertoire de la base comporte plus de 15000 sources reconnues (de genres variés couvrant divers secteurs) dont 763 sont publiées en France et 2198 en français (Europresse, <https:// nouveau.europresse.com> [consulté le 10.09.2018]).

${ }^{16}$ Nous avons décidé de limiter la période de recherche afin de reconstruire le sens le plus actuel, mais, en même temps, bien établi dans le discours de la presse écrite. 
dans des syntagmes qui reformulent ce même nom ${ }^{17}$. Cette démarche nous a permis de constituer un corpus de 450 exemples d'emploi de l'adjectif catholique(s) dans le contexte du nom Pologne. Nous avons constaté que cet adjectif apparaît dans des emplois très variés, aussi bien du point de vue structural (SAdj ou comme expansion du SN), fonctionnel (attribut, différentes figures de l'épithète), syntaxique (coordination, juxtaposition) ou des figures sémantiques (métaphores). L'examen de ces emplois nous a conduits à distinguer cinq phénomènes liés aux emplois de catholique qui contribuent à la construction du sens du nom Pologne : l'apparition de cet adjectif dans les phrases définitionnelles, sa reclassification morphosyntaxique dans le discours de presse, la modification du sens par préfixation, son fonctionnement dans les structures de prédication seconde, ainsi que les emplois métaphoriques des reformulants dont l'adjectif catholique fait partie. C'est dans cet ordre que nous présenterons les résultats de notre étude.

\section{L'ADJECTIF CATHOLIQUE DANS LES PHRASES DÉFINITIONNELLES}

L'adjectif catholique apparait, comme SAdj attribut ou comme expansion du SN attribut, dans les séquences de type métalangagier appelées phrases définitionnelles, parce qu'elles proposent une sorte de définition discursive ${ }^{18}$. Elles se caractérisent surtout par l'emploi des verbes métalinguistiques, comme par exemple : signifier, désigner, nommer, s'appeler. Selon Marie-Françoise Mortureux, ces verbes établissent une équivalence entre un reformulé (dans notre cas : le nom Pologne) et un reformulant (SAdj avec catholique ou SN dont le SAdj avec catholique fait partie). Dans le discours de presse, les verbes métalinguistiques cités ci-dessus sont rares (ou absents) à cause de leur densité métalinguistique. Dans certain cas, le discours se sert de mots moins précis et sémantiquement ambigus, comme surtout le verbe être. Celui-ci peut introduire des paraphrases discursives (mais aussi apporter d'autres prédications) ${ }^{19}$. Ainsi, dans notre corpus, les SAdj (exemple 3) et les SN en fonction d'attribut (exemples 1 et 2) comportant l'adjectif catholique sont introduits par les verbes être (exemples 1 et 3 ) et rester (exemple 2). En voilà des exemples :

(1) Pâques est une fête très importante pour les Polonais, car la Pologne est un pays catholique et le nombre des personnes pratiquantes est très élevé. (La Voix du Nord, 28.03.2010)

(2) Avec près de $90 \%$ de fidèles, la Pologne reste la nation la plus catholique du monde. (Madame Figaro [site web], 8.12.2014)

(3) La Pologne est catholique à $99 \%$, les catholiques chinois sont une toute petite minorité. (LF, 26.06.2007)

17 Dans l'analyse, nous avons dès lors pris en considération des syntagmes comme pays catholique ou bastion catholique, tous employés pour reformuler le toponyme Pologne.

18 M.-F. Mortureux, op. cit.

${ }^{19}$ Ibidem. 
La Pologne est donc définie comme un pays/une nation qui est placé(e) sous l'autorité de la religion de l'Église catholique, un pays/une nation qui professe cette doctrine. Le sens du nom propre se montre stabilisé dans les SN de type la Pologne catholique. Notre corpus d'exemples en comporte 20 occurrences $^{20}$, toute la base Europresse, 94, et le moteur de recherche Google, $34500^{21}$.

Les phrases définitionnelles contenant un SN attribut sont un outil important de catégorisation, processus cognitif fondamental. La division du monde réel au moyen de la langue est indispensable pour penser, pour manier les concepts, pour parler. Le discours de presse, qui morcèle la réalité en unités, classe la Pologne parmi les pays catholiques. Il oriente ainsi la façon de percevoir le monde et contribue à la construction du sens social de ce nom propre.

\section{LA RECLASSIFICATION MORPHO-SYNTAXIQUE DE L'ADJECTIF CATHOLIQUE}

Si l'adjectif catholique apparaît parfois sans aucune expansion dans les contextes étudiés, comme nous l'avons montré supra (exemple 1), nombreux sont les cas où cet adjectif est modifié par différentes expansions (les syntagmes prépositionnels, les adverbes, les structures comparatives) : très catholique (144 occurrences), catholique à 90\% (22 occurrences), à 90\% catholique (10 occurrences), catholique à plus de $90 \%$ (18 occurrences), à plus de $90 \%$ catholique, catholique à $95 \%$ (10 occurrences), à $95 \%$ catholique, catholique à 99\%, à 98\% catholique, catholique à $85 \%$, à majorité catholique (14 occurrences), majoritairement catholique (14 occurrences), très majoritairement catholique (2 occurrences), ̀̀ forte majorité catholique, en majorité catholique, dans sa grande majorité catholique, profondément catholique (6 occurrences), fortement catholique (2 occurrences), le plus catholique (6 occurrences), plus catholique, plutôt catholique ( 2 occurrences), de moins en moins catholique. Dans d'autres configurations encore, l'adjectif étudié entre en coordination, par exemple catholique et conservatrice (exemple 8), et se met en antéposition par rapport au nom propre. Voilà des exemples qui illustrent l'expansion de catholique par un SP (5), l'emploi avec le superlatif (4) et son antéposition (6 et 7):

${ }^{20}$ Ce nombre est multiplié dans le processus de la circulation des dires. Les expressions employées par la presse d'information sont ensuite reprises par d'autres médias (y compris la presse de commentaire, la radio et la télévision). Comme le dit Sophie Moirand, on a ici affaire à « une ronde incessante des discours du monde médiatique » (S. Moirand, op. cit., p. 158).

${ }^{21}$ Nombre de résultats obtenu le 11.09.2018, il faut cependant noter que ce dernier change quotidiennement. Nous insistons sur le fait qu'il s'agit du nombre de l'ensemble des résultats. Dans notre étude, nous ne restreignons pas les paramètres de recherche sur Google pour pouvoir montrer le degré de diffusion de certaines expressions. 
(4) La Pologne, pays le plus catholique d'Europe, voit d'un mauvais œil l'arrivée de la Madonne le jour de l'élévation de la Vierge au Ciel. (LF, 14.08.2009)

(5) Toute la Pologne, catholique à $85 \%$, pleure celui qu'elle considère comme son père spirituel et comme celui qui lui a permis de se libérer du communisme. (L, 4.04.2005)

(6) Avec ses neuf cardinaux dont six électeurs, la France se classe seconde en Europe, ex æquo avec l'Espagne, devant la catholique Pologne, mais loin derrière les trente-sept cardinaux italiens. (LF, 23.02.2006)

(7) Lors de cette visite de quatre jours, la première jamais effectuée par un patriarche de Russie dans la très catholique Pologne, le chef de l'Église orthodoxe russe doit signer avec le chef de l'Église catholique polonaise, Mgr Jozef Michalik, un appel inédit à la réconciliation polono-russe. (LM, 17.08.2012)

Le caractère morpho-syntaxique des expansions de catholique (adverbes de degré et d'intensité, les structures comparatives et le superlatif) ainsi que leur sens (les syntagmes prépositionnels exprimant une scalarité ${ }^{22}$ ) nous semblent particulièrement intéressants en raison de la sous-catégorisation grammaticale de l'adjectif catholique. Comme nous l'avons déjà remarqué, catholique est un adjectif classé comme relationnel, relatif au substantif catholicisme (cf. point 1). Par conséquent, dans le système de la langue, cet adjectif n'est pas affecté par la catégorie de degré, ne peut pas être coordonné avec les autres adjectifs, n'admet pas la fonction d'attribut et se caractérise par sa postposition par rapport au nom qu'il modifie ${ }^{23}$. Selon le TLFi, l'emploi de la gradation n'est possible que dans les tournures négatives, familières et figurées, comme des procédés pas très catholiques, un individu pas très catholique ${ }^{24}$.

Or, les emplois discursifs de l'adjectif étudié ne répondent guère à ces indications grammaticales. Dans les cooccurrences avec le nom propre Pologne et ses reformulants comme pays et nation, cet adjectif est bel et bien gradable, se coordonne avec les adjectifs qualificatifs (et relationnels), admet la fonction d'attribut ( $c f$. partie 2) et apparaît en antéposition. Nous pouvons donc dire que le discours recatégorise cet adjectif ${ }^{25}$, et il nous reste à interroger les enjeux de ce transfert. L'expression de la scalarité (la gradualité, l'expression de l'intensité et de la comparaison) est récurrente dans les emplois de catholique et présuppose l'existence d'une échelle du catholicisme. Ainsi, le degré de catholicisme des Polonais indiqué par la presse varie entre $85 \%$ et $99 \%$, mais le plus souvent, les journalistes estiment que la Pologne est « catholique à $90 \%$ » (22 occurrences

22 P. Hadermann, M. Pierrard, D. van Raemdonck, « La scalarité dans tous ses aspects », Langue Française 165, 2010, pp. 3-15.

${ }^{23}$ M. Riegel, J.-Ch. Pellat, R. Rioul, Grammaire méthodique du français, PUF, Paris 2004, p. 357 ; C. Narjoux, Le Grevisse de l'étudiant : grammaire graduelle du français, De Boeck Supérieur, Louvain-la-Neuve 2018, p. 211.

24 TLFi, définition du mot catholique.

25 Il nous semble intéressant de noter que, dans un travail sur le mot « communauté », Michelle Lecolle fait un constat similaire pour l'adjectif relationnel communautaire qui peut avoir un usage qualificatif (M. Lecolle, "Identité/altérité et noms collectifs humains. Le cas de communauté », Questions de Communication 13, 2008, pp. 323-342). 
dans la base Europresse). Remarquons que le discours ne mentionne jamais de critères qui permettraient d'indiquer le degré de scalarité (nombre de personnes baptisées, nombre de pratiquants ?). La scalarité exprimée par les SP de type à X\% en complément de l'adjectif a une portée pragmatique et s'inscrit dans la stratégie de « faire croire » que le discours est précis, exact, savant et objectif. La scalarité semble référer dans ce cas aux savoirs des sciences exactes.

Comme nous l'avons signalé supra, l'adjectif catholique, contrairement à sa nature grammaticale, est souvent coordonné et juxtaposé à d'autres adjectifs. La coordination et la juxtaposition établissent une certaine équivalence sémantique ${ }^{26}$ entre deux ou plusieurs éléments (dans notre cas c'est l'adjectif catholique et les autres adjectifs). Par conséquent, la coordination et/ou la juxtaposition contribuent à la construction du sens social du lexème catholique ${ }^{27}$. En particulier, et, qui véhicule une jonction, impose une réinterprétation du segment qui précède et spécifie qu'il existe entre le dernier élément de la série et les précédents une relation sérielle ${ }^{28}$. En voilà un exemple :

(8) La deuxième place obtenue par LPR peut confirmer en Europe le cliché d'une Pologne conservatrice, catholique et arriérée. (LF, 14.06.2004)

Dans le corpus étudié, catholique se présente accompagné des adjectifs ou groupes adjectivaux : antisémite ; arriérée ; attachée aux valeurs familiales ; blanche ; communiste ; conservatrice ; démocratique ; droitière ; économiquement faible ; europhobe; fervente ; intégriste ; millénaire ; nationaliste ; parfois fataliste; patriarcale; pratiquante; proche de cette Radio Maryja qui inquiète même le Vatican; pure; rurale; slave; solidaire des couches démunies; soucieuse de ses intérêts dans l'UE; souvent provinciale; toujours fâchée avec la Russie et avec l'Allemagne; traditionaliste; traditionnelle, ultratraditionaliste, les plus récurrents étant antisémite, conservatrice, nationaliste, traditionaliste et rurale. Une simple recherche des expressions " antisémite catholique » et «catholique antisémite » permet de générer une liste de 38 résultats dans la base de données Europresse $^{29}$ et environ 68730 résultats avec le moteur de recherche Google ${ }^{30}$. La même procédure appliquée aux autres adjectifs génère 623 (Europresse) et

${ }^{26}$ E. Biardzka, « Narzędzia badawcze francuskiej analizy dyskursu na przykładzie zdrowej żywności w dyskursie medialnym », [dans :] Kuchnia i stół w komunikacji społecznej. Tekst, dyskurs, kultura, W. Żarski, T. Piasecki (dir.), Oficyna Wydawnicza Atut, Wrocław 2017, pp. 13-21.

27 M.-F. Mortureux, op. cit.

28 J.-P. Sémon, «Les conjonctions de coordination et la construction du sens », Revue des Études Slaves 66 (3), 1994, pp. 643-668.

${ }^{29}$ Dans ces cas, nous avons tenu compte de tous les résultats, indépendamment de la période discursive, afin de rendre compte de l'ancrage de ces paires d'adjectifs dans la mémoire collective des journalistes et, par conséquent, des lecteurs.

${ }^{30}$ Toutes les données sur la fréquence des paires d'adjectifs discutés dans le paragraphe datent du 14.09.2018. 
9350 (Google) résultats pour la paire catholique + conservatrice ${ }^{31}, 544$ et 10020 résultats pour catholique + nationaliste, 1474 et 36040 résultats pour catholique + traditionaliste, 434 et 12620 résultats pour catholique + rurale ${ }^{32}$. Nos analyses montrent une relation directe, une coordination récurrente entre ce qui est catholique, rural, antisémite, conservateur, nationaliste, traditionnel. Par ces associations régulières, le sens de catholique est quasiment toujours affecté par le sens des autres lexèmes (catholique est associé à rural, antisémite, conservateur, nationaliste, traditionnel) et se grave dans la mémoire collective. La Pologne ainsi décrite est alors un pays « qui est hostile à la race juive $»^{33}$, « qui conserve, assure le maintien de quelque chose ou de quelqu'un à l'abri de toute altération physique ou morale $»^{34}$, « qui est partisan du nationalisme $»^{35}$, c'est-à-dire qui approuve le « courant de pensée fondé sur la sauvegarde des intérêts nationaux et l'exaltation de certaines valeurs nationales $»^{36}$. Le nationalisme n'est pas un courant de pensée neutre. Sur l'axe axiologique, il se situe du côté des valeurs négatives ${ }^{37}$. Le TLFi indique que le nationalisme «s'accompagne de xénophobie et/ou de racisme et d'une volonté d'isolement économique et culturel $»^{38}$ et qu'il est étymologiquement connoté de chauvinisme ${ }^{39}$. Le sens attribué au nom propre Pologne, par la proximité des adjectifs énumérés ci-dessus, renvoie aussi à l'" attachement aux valeurs, aux croyances du passé transmises par la tradition $»^{40}$. L'épithète $r u$ ral(e), quant à elle, se réfère à un territoire et un mode de vie. Dans le TLFi, cet adjectif est défini de la manière suivante : " qui vit à la campagne » et « dont l'activité s'exerce à la campagne $»^{41}$. Énumérés dans le même contexte syntagmatique, les adjectifs antisémite, conservateur, nationaliste, traditionaliste et rural sont présentés comme compatibles avec catholique. Par effet de coordination, ayant le même statut formel, ils glissent vers le même statut sémantique et forment une série. En conséquence, l'adjectif catholique, dans le discours de la presse écrite, prend un sens extrêmement négatif.

${ }^{31}$ La recherche de la paire catholique + conservateur donne 1291 résultats dans Europresse et 20010 résultats dans Google.

${ }^{32} \mathrm{La}$ recherche de la paire catholique + rural donne 84 résultats dans Europresse et 3280 résultats dans Google.

33 TLFi, définition du mot antisémite.

${ }^{34}$ Ibidem, définition du mot conservateur.

${ }^{35}$ Ibidem, définition du mot nationaliste.

${ }^{36}$ Ibidem, définition du mot nationalisme.

37 Bien que TLFi note aussi une connotation valorisante du mot nationalisme, il nous semble que la construction discursive ne met en relief que son sens péjoratif (cf. Le discours du nationalisme en Europe, G. Komur-Thilloy, A. Celle (dir.), L'Improviste, Paris 2010).

\footnotetext{
${ }^{38}$ Ibidem.

39 Ibidem.

${ }^{40}$ Ibidem, définition du mot traditionalisme.

${ }^{41}$ Ibidem, définition du mot rural.
} 
Enfin, la reclassification de l'adjectif catholique s'opère aussi par changement de place dans la phrase. Normalement postposé au nom, l'adjectif relationnel $c a$ tholique apparaît aussi en antéposition dans le discours étudié (exemples 6 et 7). La presse parle de la Pologne catholique, mais aussi de la catholique Pologne. Ce changement de position syntaxique peut impliquer un changement sémantique. En effet, dans le système de la langue française, les adjectifs à position variable prennent différents sens en fonction de la place qu'ils occupent par rapport au nom. Postposés à celui-ci, ils attribuent une simple propriété au référent du GN. Par contre, quand ils précèdent le nom, leur sens est souvent figuré ou intensifié 42 . Nous pouvons dès lors supposer que dans le discours de presse, l'antéposition de l'adjectif catholique par rapport au nom est un outil d'augmentation du sens.

\section{LA PRÉFIXATION COMME OUTIL D’AUGMENTATION DU SENS PÉJORATIF}

La valeur dépréciative attribuée à l'adjectif catholique se manifeste et se confirme par l'emploi du préfixe ultra-, qui, selon le dictionnaire, a une connotation négative (exemple 9) :

(9) Une chose est sûre, ce n'est pas dans l'Hexagone que l'on verra bientôt une ancienne playmate s'emparer d'un club de province, malgré les beaux discours de parité dont se gaussent les décideurs publics. Mais en Pologne, pays pourtant ultra-catholique, c'est possible. (Le Point.fr, 29.12.2012)

Selon le TLFi, l'épithète ultra-catholique caractérise une personne « qui observe strictement, jusqu'à l'excès, les commandements de la religion catholique $»^{43}$. En outre, l'entrée du TLFi consacrée au préfixe ultra- précise expressis verbis la valeur négative de cet affixe. En fait, ultra-suivi d'un adjectif ou parfois d'un substantif sert d'intensificateur à connotation péjorative ou laudative ${ }^{44}$. Dans les contextes d'utilisation de notre corpus, la deuxième connotation est exclue. De plus, dans le discours journalistique, le préfixe ultra- apparaît également dans le cas d'autres adjectifs importants coordonnés ou juxtaposés à catholique (voir liste ci-dessus) pour modifier le sens du nom propre Pologne (exemple 10). Ce préfixe augmente le sens négatif de l'adjectif et entre dans la logique de la coordination sur l'axe syntagmatique :

42 M. Grevisse, A. Goosse, Le Bon Usage : grammaire française, De Boeck, Bruxelles 2007, p. 413 ; M. Riegel, J.-Ch. Pellat, R. Rioul, op. cit., p. 182.

43 Ibidem, définition du mot ultra-catholique.

${ }^{44}$ Ibidem, définition du préfixe ultra-. 
(10) Un retour aux affaires d'une Pologne ultratraditionaliste et catholique, qui place sa souveraineté, mise à mal pendant des siècles par l'Allemagne et la Russie, au-dessus de tout, et aujourd'hui de Bruxelles. (Marianne, 30.10.2015)

\section{LA PRÉDICATION SECONDE ET SES EFFETS}

Comme nous l'avons indiqué dans l'introduction, l'adjectif catholique entre dans nombre de structures très hétérogènes. Pour certaines d'entre elles, on peut tout de même trouver un dénominateur commun : elles fonctionnent dans le discours de presse comme prédicats secondaires. Il s'agit de groupes nominaux en apposition ainsi que d'adjectifs dits détachés. Dans ce cas, un lien prédicatif secondaire, de type attributif, sans marque verbale de prédication, s'installe entre le thème (dans notre analyse, la Pologne) et le segment détaché ${ }^{45}$. Ce dernier se caractérise toujours par un contenu propositionnel et il est facultatif dans la phrase. À part une valeur descriptive, la prédication seconde permet de produire différents effets de sens à travers, par exemple, une fonction topique, un indice de focalisation ou du sens circonstanciel (y compris les valeurs de temps, de cause, de condition ou de concession $)^{46}$. En voilà des exemples :

(11) Toute la Pologne, catholique à $85 \%$, pleure celui qu'elle considère comme son père spirituel et comme celui qui lui a permis de se libérer du communisme. (L, 4.04.2005)

(12) Gay Pride, à $13 \mathrm{H} 00$, alors que l'homophobie reste ancrée en Pologne, pays de tradition catholique. (AFP Infos Françaises, 13.06.2014)

Le groupe adjectival «catholique à $85 \%$ », détaché par les virgules du nom Pologne auquel il se réfère, a une fonction explicative et indique la cause de l'état des choses décrit dans la prédication principale : " pleure celui [Jean Paul II] qu'elle considère comme son père spirituel et comme celui qui lui a permis de se libérer du communisme ». Nous pouvons ainsi reformuler cette phrase : «Parce que la Pologne est catholique à $85 \%$, elle pleure celui qu'elle considère comme son père spirituel $[\ldots] »$. Les groupes nominaux apposés sont liés avec le toponyme examiné par un rapport d'identité référentielle et, comme le remarquent Martin Riegel, Jean-Christophe Pellat et René Rioul, correspondent à l'attribut d'une phrase à verbe être dont le sujet serait le GN de rattachement ${ }^{47}$ (dans le cas étudié : le nom Pologne). Ainsi, en (12), la séquence " pays de tradition catholique » relève de la prédication seconde, et par sa position syntaxique, elle est mise en évidence dans la phrase.

${ }^{45}$ E. Havu, M. Pierrard, «La prédication seconde en français : essai de mise au point », Travaux de linguistique 57, 2008, pp. 7-21.

${ }^{46}$ E. Havu, M. Pierrard, « Détachement et prédication seconde », Neuphilologische Mitteilungen CVIII/4, 2007, pp. 729-742.

${ }^{47}$ M. Riegel, J.-Ch. Pellat, R. Rioul, op. cit., p. 190. 
(13) Pour la Pologne, pays à 90\% catholique, le réveillon est, en effet, une institution sacrée assaisonnée à la carpe. (L, 23.12.2004)

(14) La législation en Pologne, pays à $95 \%$ catholique, interdit toute forme de conservation des fœetus et des corps des morts-nés. (AFP Infos Mondiales, 20.10.2005)

(15) En Pologne, pays conservateur, catholique, patriarcal, le sujet est tabou, la famille est sacrée, va à l'église, et ce qui se passe au sein de la cellule familiale ne concerne personne d'autre. (L, 8.04.2017)

Les séquences de prédication seconde comprenant l'adjectif catholique n'ajoutent pas seulement un trait catholique au sens du nom propre Pologne. Par leur visée explicative, elles font du catholicisme une cause des comportements des Polonais, ou - au sens plus large - un marqueur de la vie sociale en Pologne.

\section{LES MÉTAPHORES DE LA GUERRE : LA POLOGNE, BASTION CATHOLIQUE}

Après avoir analysé les structures morpho-syntaxiques, penchons-nous sur un autre processus participant à la construction du sens du nom propre Pologne : la métaphore. La métaphore n'est pas seulement une figure de style propre au discours littéraire, elle est bel et bien présente dans les pratiques langagières quotidiennes. Comme l'ont remarqué George Lakoff et Mark Johnson, il n'est pas possible de se passer de la métaphore car elle est indispensable au processus de la pensée et, par conséquent, à la langue ${ }^{48}$. Depuis leurs études, en linguistique cognitive, la métaphore est décrite comme une projection sélective des traits d'un domaine conceptuel sur un autre. En tant que « figure par laquelle on désigne un référent en utilisant un autre signe que celui qui le désigne couramment, par une comparaison sous-entendue $»^{49}$, la métaphore sert à saisir ce qui ne peut être saisi autrement, elle " parle » à l'imagination ${ }^{50}$. Les linguistes représentants de l'approche praxématique, basée sur le concept de la construction des sens dans le discours, précisent que l'actualisation métaphorique impulse une intentionnalité : elle reflète la façon dont le sujet perçoit le monde ${ }^{51}$. Les sens ainsi créés constituent des sens communs, partagés par toute la communauté dans laquelle ils ont été produits. L'emploi de métaphores capte l'attention du lecteur et l'aide à se faire une idée du sujet décrit.

Au fil de la production médiatique, on peut observer la récurrence de métaphores guerrières, et notre corpus ne fait pas exception à ce phénomène. Comme

48 G. Lakoff, M. Johnson, Metafory w naszym życiu, trad. T.P. Krzeszowski, Aletheia, Warszawa 2010, p. 29.

${ }^{49}$ P. Charaudeau, D. Maingueneau, Dictionnaire d'analyse du discours, Seuil, Paris 2002, p. 35 .

${ }^{50}$ Ibidem, p. 36

${ }^{51}$ C. Détrie, Du sens dans le processus métaphorique, Honoré Champion, Paris 2001, p. 162. 
le montre Charlotte Dilks dans son étude d'inspiration cognitive, l'écriture journalistique est pleine de vocables projetant des traits du domaine de la guerre sur le domaine cible (dans le cas étudié : discours sur la Pologne comme pays catholique). Il s'agit surtout des verbes (affronter, attaquer, bombarder, conquérir, défendre...) mais aussi de noms et d'adjectifs ${ }^{52}$. Par leur fréquence importante dans le discours de presse, ce type d'expressions, presque lexicalisées, ne sont plus perçues comme figurées et se rapprochent des métaphores conventionnalisées (figées) dont le sens n'est plus calculé mais simplement repris dans le lexique mental. La métaphore conventionnalisée ne renvoie que faiblement à son domaine source. Par exemple, dans Une guerre des prix, l'expression est devenue stéréotypée ${ }^{53}$.

Cependant, la métaphore conventionnalisée peut véhiculer des effets de sens particuliers en fonction de nouveaux contextes. En ce qui concerne notre corpus, parmi tous les reformulants introduits par le métalangage, nous en avons rencontré qui mettent en relief le statut de défenseur du catholicisme attribué au nom Pologne. Dans le discours, ce toponyme ne désigne pas seulement un pays catholique. Sur le mode métaphorique, il fait référence à un combattant qui défend les intérêts de l'Église catholique. On peut induire ce sens d'expressions telles que bastion catholique (exemple 16), forteresse catholique (exemple 17), ou encore d'une séquence moqueuse, bons petits soldats catholiques, qui fonctionne ici dans une simple comparaison construite avec comme (exemple 18) :

(16) En fait, l'onde de choc ressentie mardi à Rome revient comme un boomerang des ÉtatsUnis où les catholiques sont très organisés pour la défense de la vie, d'Afrique où ces préoccupations occidentales apparaissent surréalistes, mais aussi de bastions catholiques comme la Pologne qui n'admet pas une telle volte-face romaine après trois décennies d'enseignements de Jean-Paul II et Benoît XVI. (LF, 15.10.2014)

(17) On sait bien vite qu'il vient de Pologne, ardente forteresse catholique fichée en terre communiste telle une croix d'exorciste dans le royaume de l'Antéchrist derrière le rideau de fer. (L, 2.04.2005)

(18) Mais si le Portugal, l'Espagne, la France, l'Italie et d'autres bons petits soldats catholiques comme la Pologne et le Luxembourg ont appliqué la réforme grégorienne sur-le-champ, les pays protestants et orthodoxes ont traîné des pieds. (L, 15.04.2016)

Ainsi reformulé, le nom Pologne gagne le sens de lieu de résistance décrit par le TLFi dans la première entrée du mot bastion : " ouvrage de fortification, généralement de forme pentagonale, faisant partie de l'enceinte du corps d'une place, présentant en saillie deux flancs et deux faces, fait d'un gros amas de terre soutenu de muraille, de gazon ou de terre battue ${ }^{54}$. Dans un sens figuré, le mot

${ }^{52} \mathrm{Cf}$. Ch. Dilks, Les métaphores de guerre dans la prose journalistique du français, Institutionen för franska, italienska och klassiska språk, Stockholm 2009.

${ }^{53} \mathrm{Ch}$. Dilks, « Approches théoriques : la métaphore, la sémantique interprétative et la sémantique cognitive ", Texto! Textes \& Cultures XVI/2, 2011, <http://www.revue-texto.net/index. php?id=2857> [consulté le 3.11.2018].

54 TLFi, définition du mot bastion. 
bastion peut également désigner « ce qui constitue un moyen de résistance ou de défense $»^{55}$. Le mot forteresse induit un sens similaire. Il se réfère à un « lieu fortifié de plus ou moins grande étendue, destiné à défendre une place ou une région » ${ }^{56}$ ou, au figuré, à « ce qui enferme, défend, protège avec force quelqu'un ou quelque chose, le rend inaccessible ou inaltérable $»^{57}$. Cette dernière définition fournit une information supplémentaire sur le sens de la Pologne en tant que forteresse catholique et sur le catholicisme en tant qu'objet de la protection. Premièrement, la Pologne, qui est forteresse, semble s'approprier le catholicisme et le rendre inaccessible aux autres. Deuxièmement, il s'agit de défense du catholicisme sous sa forme intacte, non modifiée depuis des décennies. Ces propriétés sont évoquées dans l'exemple (17) où la Pologne est comparée à une croix d'exorciste destinée à chasser les démons présents dans ce royaume de l'Antéchrist.

La résistance aux changements dans le domaine religieux semble aussi être présupposée par le verbe rester employé afin d'établir une équivalence entre le nom propre Pologne et ses paraphrases (exemple 2). Le TLFi définit ce verbe ainsi : « continuer d'être de façon plus ou moins prolongée ou durable, dans un lieu ou dans un état $»^{58}$. La Pologne est définie comme un pays qui garde ses traditions catholiques. Étant donné la valeur négative attribuée à l'adjectif catholique dans le discours médiatique étudié, si le nom Pologne désigne, sur le mode métaphorique, un défenseur du catholicisme, il désigne également un défenseur de quelque chose de mauvais. Les différents reformulants du toponyme lui imputent le sens d'un pays qui, malgré l'évolution du monde, reste fidèle à des valeurs négatives et les protège farouchement.

\section{CONCLUSION}

À une époque post-séculière, par l'emploi de l'adjectif catholique dans le contexte du nom Pologne, le discours de la presse quotidienne évoque des sens et des valeurs toujours importants du point de vue social. L'emploi de cet adjectif relationnel va au-delà de la simple attribution des traits et dépasse les usages prévus dans les grammaires et dans les dictionnaires. Rappelons brièvement le comportement discursif de l'adjectif catholique: (1) il fait partie des SN attributs et participe ainsi à la catégorisation du nom auquel il se réfère ; (2) il se comporte en véritable adjectif qualificatif (scalarité, coordination, antéposition). Par coordination, il emprunte un sens négatif qui peut être augmenté par l'antéposition et à travers l'expression d'une scalarité ; (3) les syntagmes détachés et apposés dont il fait partie fonctionnent comme prédicats secondaires et ont surtout une fonction

\footnotetext{
55 Ibidem.

${ }^{56}$ Ibidem, définition du mot forteresse.

57 Ibidem.

${ }^{58}$ Ibidem, définition du mot rester.
} 
explicative indiquant la cause des phénomènes et des événements décrits ; (4) il entre ensuite en différentes relations lexicales sur le mode métaphorique.

L'ensemble de ces pratiques discursives révèle une sorte de stratégie qui conduit à la construction du sens social de l'adjectif et qui met en avant son sens péjoratif. À l'opposé de l'avis des dictionnaires qui n'affichent aucun qualificateur axiologique, dans le discours de presse, catholique n'est pas un adjectif neutre. Au contraire, converti en adjectif qualificatif, il est avant tout un outil de jugement. $\mathrm{Si}$, par l'effet de sa coordination avec d'autres adjectifs, catholique incarne le mal dans le monde civilisé (antisémite, arriérée, nationaliste), la scalarité permet d'accentuer encore cette valeur : sur l'échelle de ce qui est catholique (qui est donc échelle du mal), la Pologne catholique se place en haut, là où le mal existe et est extrême. Le marquage axiologique s'observe aussi à travers la préfixation (ultra- devant l'adjectif). Le tableau reste cohérent avec l'antéposition (la très catholique Pologne), les effets de la prédication seconde, et les reformulants métaphoriques (le bastion catholique) : la Pologne n'est rien d'autre que le gardien de dogmes et de traditions contestables.

\section{PAYS, BASTION AND FORTERESSE CATHOLIQUE? CONSTRUCTION OF THE MEANING OF THE PROPER NAME POLOGNE IN PRESS DISCOURSE}

Summary

This paper is part of a broader study on the social meaning (sens social) of the proper name Pologne (Poland) created in the media production. The analysis of press discourse makes it possible to observe the recurrence of the adjective catholique (Catholic) in the co-occurrences of this toponym. The aim of this paper is, firstly, to examine the syntactic entourage of the name Pologne in which the adjective catholique appears and, secondly, to analyse, on the paradigmatic axis, reformulations of the toponym Pologne where this adjective appears (in the diaphoric network). This approach results in the description of the micro-field of the lexico-discursive profile of the name Pologne.

Key words: discourse, discourse analysis, social meaning, Poland, Catholicism. 